\title{
ESE-1 is Required to Maintain the Transformed Phenotype of MCF-7 and ZR-75-1 Human Breast Cancer Cells
}

\author{
Darius M. Walker ${ }^{1}$, Joanna M. Poczobutt ${ }^{1}$, Melissa S. Gonzales ${ }^{2,4}$, Henrick Horita ${ }^{3}$ and \\ Arthur Gutierrez-Hartmann ${ }^{*}, 1,2$
}

Molecular Biology Program ${ }^{1}$, and Departments of Medicine ${ }^{2}$, Biochemistry and Molecular Genetics ${ }^{3}$ and
Pharmacology ${ }^{4}$, University of Colorado Denver, Anschutz Medical Center, Aurora, CO, 80045, USA

\begin{abstract}
Background: ETS factors comprise a large transcription factor family known to play a significant role in cellular development, differentiation, and transformation. The human Epithelial Specific Ets factor-1, ESE-1, is particularly relevant in breast cancer. Specifically, increased mRNA expression of ESE-1 and the Her2/neu protooncogene are correlated in breast cancer, and activation of the Her2/Neu receptor induces ESE-1 gene transcription. Stable expression of ESE-1 initiated transformation of ESE-1-negative MCF-12A immortalized human mammary epithelial cells, leading to increased migration, invasion and anchorage independent growth. However, little is known about ESE-1 protein expression and its role in maintaining the transformed phenotype in human breast cancer cell lines. Results: Here, we used an anti-ESE-1 mouse monoclonal antibody in Western blot and immunofluorescent cell analyses to show that ESE-1 is expressed as a nuclear protein in MCF-7, T47D and ZR-75-1 transformed, tumorigenic mammary epithelial cell lines, and that it is not expressed in transformed MDA-MB-231 and nontransformed MCF-10A and MCF-12A cells. In addition, specific knockdown of endogenous ESE-1 in the human breast carcinoma ZR-75-1 and MCF-7 cell lines decreased colony formation and anchorage independent growth. Mechanistically, ESE-1 knockdown decreased cellular proliferation, but had no effect on apoptosis. Conclusions: These results establish that the knockdown of a single ETS factor, ESE-1, is sufficient to reverse the transformed phenotype in breast cancer and demonstrate that ESE-1 is required for cellular proliferation. Thus, ESE-1 plays a key role in maintaining the transformed phenotype in breast cancer, providing a novel single-point target for therapy.
\end{abstract}

Keywords: ETS, transformation, breast cancer, shRNA.

\section{INTRODUCTION}

The ETS transcription factor family is composed of 27 members in humans, and ETS proteins appear to have important roles in cellular proliferation, differentiation and transformation [1-3]. This large family of transcription factors is characterized by a conserved winged helix-turnhelix DNA binding domain (DBD), the ETS domain, which mediates binding to target DNA sequences [3]. ETS proteins function as transcriptional activators or repressors, and are regulated by protein-protein interactions and mitogenactivated protein kinase (MAPK) phosphorylation [1-3]. In particular, activation of the Ras proto-oncogene has been shown to mediate phosphorylation of several ETS factors [3, 4]. This is significant because the Ras pathway is critical in regulating cell cycle and proliferation $[3,4]$. This, in conjunction with ETS chromosomal translocations and overexpression, suggests that this family has a major role in oncogenesis $[1,5,6]$.

Epithelial specific ETS factor-1, ESE-1 (also known as ESX, Jen and ERT, and Elf3 in mice) is a $\sim 42 \mathrm{kDa}$ protein, and it is the defining member of the epithelial-restricted, ESE subfamily of ETS transcription factors [7-10]. The

*Address correspondence to this author at the Molecular Biology Program, and Departments of Medicine, University of Colorado Denver/AMC, P. O. Box 6511, Mail Stop 8106, Aurora, CO 80045, USA; Tel: 303-724-3921; Fax: 303-724-3920; E-mail: a.gutierrez-hartmann@UCHSC.edu
ESE-1 cDNA was first isolated while screening cDNA from human keratinocytes and pancreatic cancer tissue for novel transcripts containing the ETS domain [7, 9]. Since ESE-1's initial isolation, its mRNA expression has been documented in several human and rodent epithelial tissues, including placenta, lung, kidney, prostate, intestine, breast, skin, retina and other epithelia [7-10]. During mouse embryo development, Elf3 mRNA expression levels increase progressively, from embryonic day 7 to day 17 , and in post-embryonic mammary gland development, Elf3 is found in virgin, pregnant and involuting mammary glands [11]. The ESE-1 locus, 1q31.1, is located in a region that is commonly amplified in human breast cancer $[10,12]$. Furthermore, over-expression of ESE-1 mRNA has been demonstrated in lung cancer and in Her2/neu-positive ductal carcinomas in situ (DCIS) [10, 12]. This increased ESE-1 mRNA expression has been shown to correlate with increased expression of the $\mathrm{Her} 2 / \mathrm{Neu}$ proto-oncogene in breast cancer cell lines and in 45 primary ductal breast cancer samples $[12,13]$. In addition, activation of the Her2/Neu receptor induces ESE-1 gene transcription $[12,13]$. Therefore, a positive-feedback-loop is predicted based on increased ESE-1 expression in response to Her2/neu receptor activation and ESE-1 protein then binding and activating the Her2/neu promoter [13-16].

An increasingly large body of evidence reveals that ETS factors are particularly important in breast cancer $[1,5]$. A thorough mRNA expression analysis of 25 ETS factor members in normal and cancerous mouse mammary glands sho- 
wed that expression of Pdef, Pea3, Elf3/ESE-1, Elf5/ESE-2, Ehf/ESE-3, ETV6/TEL, and Elf2/NERF mRNAs was elevated in the epithelial cell compartment of mammary tumors [17]. Moreover, expression of a dominant-negative ETS, to overcome the redundancy of ETS factor expression, reversed the transformed phenotype in NmuMG, MMT and BT20 breast cancer cell lines $[18,19]$. To determine whether a single ETS factor could impose the transformed phenotype, we stably expressed HA-ESE-1 or GFP-ESE-1 fusions in the ESE-1-negative, non-transformed MCF-10A and MCF-12A human mammary cell lines, and demonstrated that ectopic ESE-1 increased cellular proliferation, migration, invasion and colony number in soft agar [20,21]. Moreover, ESE-1 was identified as a factor enhancing cell migration and altered morphogenesis in 3D assays in a separate and unbiased analysis of a collection of 1000 cDNAs relevant to breast cancer, in which each of 1000 cDNAs were ectopically expressed in MCF-10A cells [22]. Finally, we have reported that ESE-1 initiates transformation of MCF-12A mammary epithelial cells via an autonomously functioning, unique 40-amino acid serine and aspartic rich (SAR) domain acting via a novel cytoplasmic mechanism [20]. While dominant-negative and gain-of-function experiments demonstrate that ETS factors, in particular ESE-1, mediate the transformed state in breast cancer, no study to date has demonstrated that ESE-1 alone is required to maintain the transformed phenotype.

To investigate the potential role of ESE-1 in maintaining a malignant phenotype, we used shRNA targeting ESE-1 and a highly-specific ESE-1 mouse monoclonal antibody (Walker et al., in preparation), in order to characterize and monitor ESE-1 protein expression in several nontransformed and transformed mammary epithelial cell lines. Here we show that shRNA targeting ESE-1 specifically ablates endogenous ESE-1 expression in ZR-75-1 and MCF-7 cells, leading to an inhibition of clonogenicity and anchorage independent colony growth in these cells. Moreover, we show that ESE-1 knockdown did not induce apoptosis, but rather diminished MCF-7 cellular proliferation. These results establish that ESE-1 plays a critical role in maintaining the transformed state and that it does so by controlling cell proliferation. Thus, ESE-1 provides a potential single-point target for future breast cancer therapy.

\section{MATERIALS AND METHODOLOGY}

\section{Cell Lines}

MCF-7, T47D, MDA-MB-231, MCF-10A, and MCF$12 \mathrm{~A}$ cells were maintained as described previously $[15,21]$. ZR-75-1 cells were cultured in minimum essential medium (MEM) supplemented with 5\% FBS, 1x non-essential amino acids, and $1 \mu \mathrm{M}$ insulin. For soft agar experiments, ZR-75-1 cells were cultured in RPMI 1640 medium supplemented with $10 \%$ FBS.

\section{shRNA Constructs and Transfection}

ESE-1 shRNA and shCtr oligonucleotides were designed using Oligoengine (Seattle, WA). The oligonucleotide targeting ESE-1 (shESE-1) is follows: forward oligo 5' ACAGC AACATGACCTACGATTCAAGAGATCGTAGGTCATG TTGCTGT-reverse oligo 5' ACAGCAACATGACCTACG
ATCTCTTGAATCGTAGgTCATGTTGCTGT. The ESE-1 negative control shRNA oligonucleotide is as follows: forward oligo 5' GCTCAACGAGGGCCTCATGTTCAAG AGACATGAGGCCCTCGTTGAGC reverse oligo 5' GCTC AACGAGGGCCTCATGTCTCTTGAACATGAGGCCCTC GTTGAGC. This negative control (shCtr) was originally designed to target ESE-1 expression, but since qRT-PCR and Western blot studies showed it failed to inhibit ESE-1 expression, we used it as a negative control. The ESE-1 shRNA and shCtr were cloned into pSuper at the $B g I I I$ and HindIII restriction sites. Each pSuper shRNA vector was cotransfected along with pEGFP-C3 at a 10 to 1 ratio (pSuper: pEGFP) into MCF-7 or ZR-75-1 cells using Qiagen Effectene, with a 12 to 1 ratio of DNA to Effectene. Cell lysates were generated two days post-transfection, unless otherwise noted. The shESE-1/ob4 construct was identified as capable of reducing endogenous ESE-1 protein, after testing an additional five shESE-1 constructs in the pLKO.1 lentiviral plasmid backbone, which contains a puromycin expression cassette. A single shGFP in the pLKO.1 vector served as a negative control. All of these pLKO.1 shRNAs were purchased from Open Biosystems (Huntsville, AL) and transfected as plasmid DNAs, not as packaged lentiviruses. The oligonucleotide targeting ESE-1 (shESE-1/ob4) sequence targets the TAD and is as follows: 5'-GCTCTTC TGATGA GCTCAGTTG. The GFP negative control shRNA oligonucleotide is as follows 5'-TACAACAGCCACAACG TCTAT. Each pLKO.1 shRNA vector was co-transfected along with pEGFP-C3 at a 10 to 1 ratio (pLKO.1:pEGFP) into MCF-7 cells using Qiagen Effectene, with a 12 to 1 ratio of DNA to Effectene. Cell lysates were generated two days posttransfection, as noted above.

\section{Western Blotting}

Western blot analysis was performed essentially as described previously $[15,20]$, but cells lysis was completed by re-suspending them and incubating them on ice for $25 \mathrm{~min}$ in $0.1 \% \mathrm{NP}-40,50 \mathrm{mM}$ Hepes pH $7.2,250 \mathrm{mM} \mathrm{NaCl}, 2 \mathrm{mM}$ EDTA, $50 \mathrm{mM} \mathrm{NaF}, 1 \mathrm{mM} \mathrm{Na} \mathrm{VO}_{4}, 1 \mathrm{mM}$ PMSF, $1 \mathrm{mM}$ benzamidine, $1 \mathrm{mM}$ DTT, $25 \mu \mathrm{g} / \mathrm{ml}$ aprotinin, $25 \mu \mathrm{g} / \mathrm{ml}$ trypsin inhibitor, $25 \mu \mathrm{g} / \mathrm{ml}$ leupeptin, and $25 \mu \mathrm{g} / \mathrm{ml} \beta$ glycerophosphate. Cells were then vortexed in four 30 second intervals, followed by a $15 \mathrm{~min}$ centrifugation at $13,000 \mathrm{~g}$ at $4^{\circ} \mathrm{C}$, and the supernatant was collected. Protein concentrations were determined using a Bio-Rad detergentcompatible protein assay.

\section{Quantitative RT-PCR}

The qRT-PCR study was performed as described [23]. Plasmid pEGFP-ESE-1 [20] was used to generate sensestrand ESE-1 RNA, which was then used as an absolute standard for qRT-PCR. Primers and probes for ESE-1 were designed using the Prism 7700 sequence detection software (Primer Express, Perkin-Elmer Corp./Applied Biosystems (PE ABI), Foster City, CA), resulting in the following oligos: Forward ESE-1 Primer: (1109)AGCCGGGCCATGA GGTAC(1126); Reverse Primer: (1173)ACGAGTCGCCGG CCAT(1158); and TaqMan Probe: (1131)ACAAACGGGAG ATCCTGGAACGGG(1154). Total RNA was prepared from cells using RNA Stat-60 (Tel-Test "B", Inc.) and qRT-PCR was performed using an ABI PRISM 7700 Sequence detector 
(PE ABI), with PCR reactions monitored in real time. Reverse transcription, PCR conditions, real-time data acquisition and analyses were performed as described [23]. ESE-1 mRNA was normalized to the amount of $18 \mathrm{~s}$ rRNA (PE ABI, $\mathrm{P} / \mathrm{N}$ 4308310) in each sample.

\section{Immunocytochemistry (ICC)}

Cells $(50,000)$ were plated directly onto glass cover slip in a 12 well tissue culture plate. Two days post-plating, cells were fixed with $2 \%$ paraformaldehyde (PFA) in 1x PBS for 20-25 min at room temperature (RT), followed by three 5 min washes in $1 x$ PBS. Cells were permeabilized at RT with $0.5 \%$ Triton $\mathrm{X}-100$ in $1 \mathrm{x}$ PBS for 10 minutes, followed by three $10 \mathrm{~min}$ washes in $100 \mathrm{mM}$ glycine in $1 \mathrm{x}$ PBS. Permeabilized cells were blocked in a $1 \mathrm{x}$ PBS, 0.5\% Tween-20, $10 \%$ goat serum, $0.05 \%$ bovine serum albumin (BSA) blocking buffer at RT within a moisture chamber for 1-2 h. Cells probed for ESE-1 were incubated in 1:500 antibody:blocking buffer overnight at $4^{\circ} \mathrm{C}$ in a moisture chamber. To measure auto-fluorescence, cells were incubated overnight at $4^{\circ} \mathrm{C}$ with blocking buffer alone. shRNA tranfected cells underwent the same procedure at $48 \mathrm{~h}$ post-transfection.

\section{Colony Formation Assays}

MCF-7 and ZR-75-1 cells were transfected in suspension with Effectene, as described above, using 250,000 cells and 800:80 ng of shRNA:pEGFP-C3 in sterile $1.5 \mathrm{ml}$ microfuge tube per transfection. Transfected cells (500,000 MCF-7 and 250,000 ZR-75-1) were then seeded on $60 \mathrm{~mm}$ plates, and $48 \mathrm{~h}$ post-transfection, cells were treated with $500 \mu \mathrm{g} / \mathrm{ml}$ G418 (Gibco) for 14 days. On day fourteen, cells were fixed with $2 \%$ PFA for $30 \mathrm{~min}$, washed twice in PBS, and then stained for 30 seconds with crystal violet and washed for 1 min in $\mathrm{ddH}_{2} \mathrm{O}$. Colonies were counted by direct visual analysis.

\section{Colony Formation in Soft Agar}

Anchorage independent growth was determined by assaying colony formation in soft agar. MCF-7 or ZR-75-1 cells were transfected with shESE-1 or shCtr plasmids and 50,000 cells were seeded by re-suspending cells in $1.5 \mathrm{ml}$ per well of $0.3 \%$ agar noble (BD Scientific Difco) mixed with growth medium, and plated in 6 well plates previously covered with $1.5 \mathrm{ml}$ of $0.6 \%$ agar noble base layer per well. Optimal colony formation by ZR-75-1 cells required additional modifications, including switching to RPMI 1640 medium supplemented with $10 \%$ serum and concentrating the medium, so that the concentration of nutrients was $1 \mathrm{x}$ after mixing with the agar. MCF-7 and ZR-75-1 cells grown in soft agar cultures were fed every three days up to 14 days. Resulting colonies were stained overnight at $37^{\circ} \mathrm{C}$ with 150 $\mu \mathrm{l}$ of $1 \mathrm{M}$ nitroblue tetrazolium chloride (Amresco) in PBS and quantitated using Metamorph or ImageJ imaging software, set to a colony threshold size of 150 microns.

\section{Apoptosis Assay}

MCF-7 cells were transiently transfected with empty vector, shCtr or shESE-1 DNA $\left(\sim 1 \times 10^{6}\right.$ cells per DNA) and used for both the DNA laddering and caspase 3/7 assays. For the DNA laddering, transfected cells were harvested $24 \mathrm{~h}$ post-transfection, cells were counted and genomic DNA was isolated from 100,000 cells. DNA was analyzed on a $1 \%$ agarose gel stained with ethidium bromide. Positive control cells were treated with $75 \mu \mathrm{g} / \mathrm{ml}$ of TRAIL plus $1 \mu \mathrm{g} / \mathrm{ml}$ of cycloheximide. For the Caspase-Glo $3 / 7$ assay transfected MCF-7 cells were collected $24 \mathrm{~h}$ post-transfection with $1 \mathrm{x}$ PBS/EDTA, counted and 3,000 transfected cells were plated in a 96-well plate. Caspase $3 / 7$ reagent was added to cells at $48 \mathrm{~h}$ and $72 \mathrm{~h}$ time points, and assays were performed as described by the manufacture (CaspaseGlo 3/7 Assay, Promega).

\section{Proliferation Assays}

Total cell count proliferation assays were conducted by plating 50,000 cells/well in a 12-well plate, and transfecting these cells with pEGFP-C3 and the indicated shRNA. Cells were harvested with $1 \times$ PBS/EDTA on days 2, 4, and 6 posttransfection, and viable cells were counted using the Vi-Cell counter (Beckman Coulter). MTS proliferation assays were conducted by transfecting MCF-7 cells with pEGFP-C3 and the indicated shRNA, and harvested $24 \mathrm{~h}$ later by suspension into $1 \mathrm{x}$ PBS-EDTA. Viable cells were counted by staining cells with Trypan blue and using a Vi-Cell-cell viability analyzer (Beckman Coulter), with 5,000 cells/well plated in a 96-well plate. Six days later, cells were counted using the MTS proliferation assay, as described by manufacturer (Promega).

\section{RESULTS}

MCF-7, T47D and ZR-75-1 Human Breast Cancer Cells Express ESE-1 Protein, whereas Transformed MDAMB-231 and Nontransformed MCF-10A and MCF-12A Cells do not

In order to characterize and monitor ESE-1 protein expression in basal and shRNA knockdown conditions in various human mammary epithelial cell lines, we generated several highly specific mouse monoclonal antibodies against an ESE-1 peptide spanning amino acids 128-259. This region includes the transcription activation domain (TAD), SAR, and AT-hook domains. Details describing the generation and characterization of these antibodies will be presented elsewhere (Walker DM, in preparation), but in this report we limited our studies to the use of one of these, anti-ESE-1 mAB405. Western blot analysis of whole cell extracts probing for endogenous ESE-1 was performed on a series of human transformed and nontransformed mammary epithelial cell lines and compared with qRT-PCR. As shown in Fig. (1A), ESE-1 protein was not detected by Western blot analysis in the nontransformed MCF-10A and MCF-12A mammary epithelial cells lines. In contrast, ESE-1 protein was detected in the tumorigenic MCF-7, T47D and ZR-75-1 cell lines, with the levels in MCF-7 and T47D being equivalent and greater than that expressed in ZR-75-1. Noteworthy, ESE-1 protein was not detectable in the highly metastatic MDA-MB-231 cells (Fig. 1A). In order to obtain a direct comparison of ESE-1 protein and mRNA levels in this same panel of mammary epithelial cell lines, we next performed a quantitative RT-PCR analysis. This qRT-PCR study revealed that MCF-10A and MCF-12A nontransformed cells do not express any detectable ESE-1 mRNA, 
whereas the transformed MCF-7, T47D, ZR-75-1 and MDAMB-231 cells all express ESE-1 mRNA, but to varying degrees (Fig. 1B). The MCF-10A, MCF-12A and MCF-7 mRNA and protein data do correlate with each other (Fig. 1).

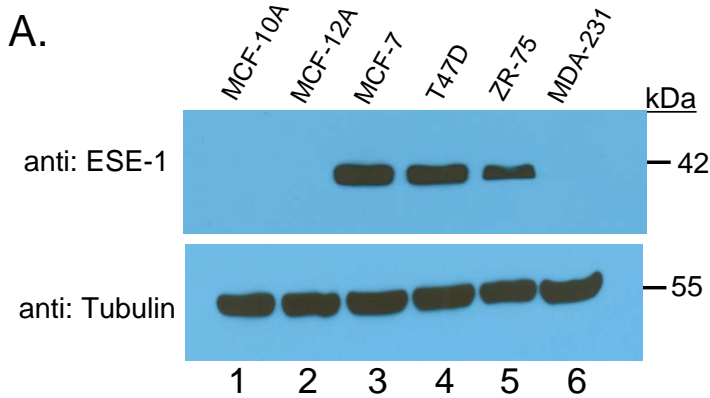

B.

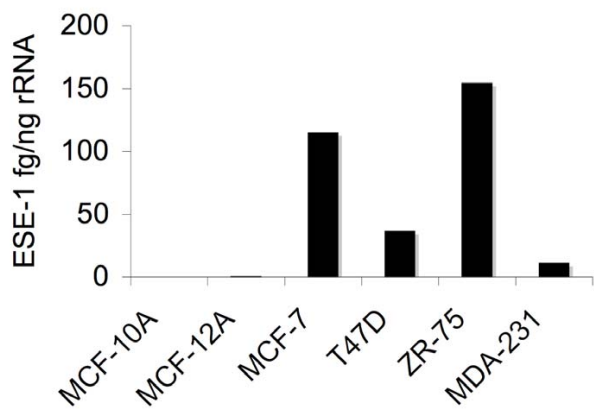

Fig. (1). ESE-1 protein is expressed in several human breast cancer cell lines. A. Western blot of whole cell extracts $(100 \mu \mathrm{g})$ generated from MCF-10A (lane 1), MCF-12A (lane 2), MCF-7 (lane 3), T47D (lane 4), ZR-75-1 (lane 5) and MDA-MB-231 (lane 6) cells, probed with anti-ESE-1 mAB405 antibody (1:1000 dilution) and anti-tubulin mouse monoclonal antibody $(1: 10,000$ dilution, CP06, Calbiochem). B. Quantitative RT-PCR analysis of endogenous ESE-1 in MCF-10A, MCF-12A, MCF-7, T47D, ZR75-1 and MDA-MB-231 human mammary cell lines. Total RNA (1 $\mu \mathrm{g})$ was generated from each cell line and used for qRT-PCR analysis (ABI PRISM 7700, PE ABI). ESE-1 mRNA in each sample calculated relative to absolute ESE-1 values that were derived from a standard curve, using a known amount of sensestrand ESE-1 RNA, and then normalized to total input RNA, using $18 \mathrm{~S}$ rRNA measured from $1 \mathrm{ng}$ total RNA.

However, for each given amount of ESE-1 protein expressed, the T47D mRNA level is lower than expected, whereas the ZR-75-1 and MDA-MB-231 mRNA levels are higher than expected (Fig. 1). To further confirm ESE-1 protein expression levels and its subcellular localization, we performed indirect immunofluorescence cytochemistry (ICC) studies, with cell nuclei counterstained with DAPI to define each cell (Fig. 2). These data revealed that MCF-10A, MCF-12A and MDA-MB-231 cells fail to express any ESE1 protein detectable by this ICC method, whereas endogenous ESE-1 protein was detected in transformed MCF-7, T47D and ZR-75-1, and in each case ESE-1 was localized to the nucleus (Fig. 2). However, the ICC and Western blot data did not strictly correlate, since T47D cells displayed the strongest ICC signal, yet in the Western blot, the T47D signal was equivalent to the MCF-7 lane, but more than the ZR-75-1 lane (Figs. 1 and 2). As a negative control, primary anti-ESE-1 monoclonal antibody was omitted for each cell line, and this study revealed that the ICC signal is dependent on the primary anti-ESE-1 antibody (Fig. 2, lower panel). In general, the ICC data further confirmed the Western blot data and revealed ESE-1 protein to be primarily localized in the nucleus.

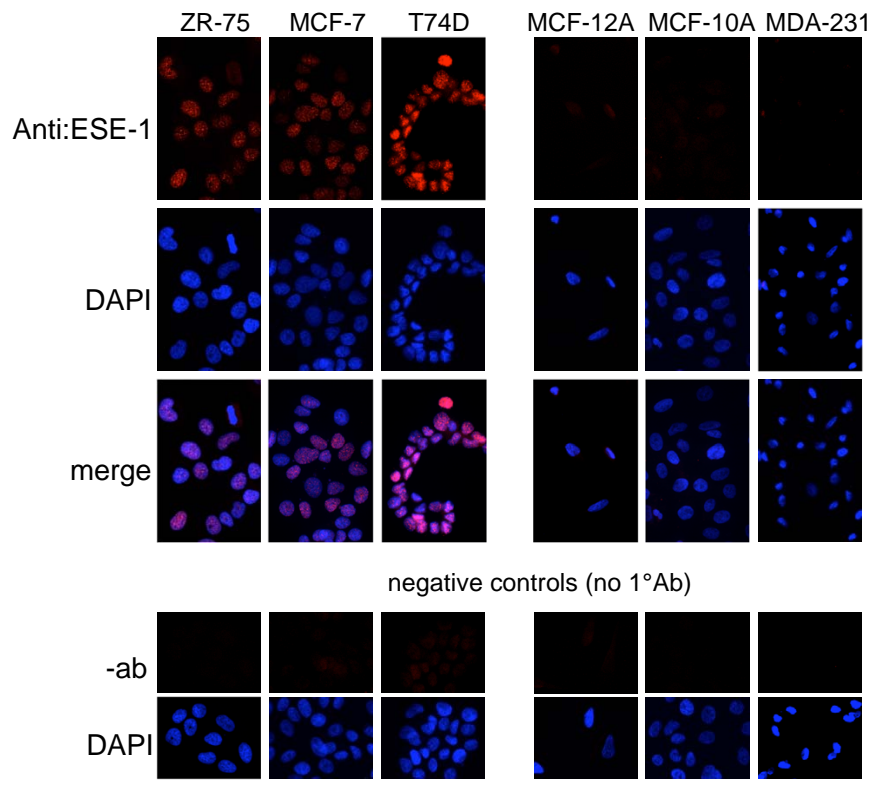

Fig. (2). ESE-1 is localized to the nucleus in human breast cancer cell lines. The top row are confocal images of ZR-75-1, MCF-7, T47D, MCF-12A, MCF-10A and MDA-MB-231 mammary epithelial cells probed with the anti-ESE-1 mAB405 antibody (1:500 dilution), followed by a Cy3-conjugated goat antimouse secondary antibody (1:200 dilution). ZR-75-1, MCF-7, T47D show a positive (red) signal and are grouped in the left panel, whereas MCF-12A, MCF-10A and MDA-MB-231 are negative and are grouped in the right panel. The second row from the top shows confocal images of the same cells stained with DAPI $(100 \mathrm{ng} / \mathrm{ml}$; blue nuclei), and the third row shows the merge of the top two rows. Shown at the bottom are the negative controls, depicting confocal images of these same cells probed with blocking solution and the Cy3-conjugated goat anti-mouse secondary antibody (1:200 dilution), but omitting the anti-ESE-1 mAb. The bottom row shows confocal images of DAPI-stained, negative control cells.

\section{shRNA Targeting of ESE-1 Knocks Down Endogenous ESE-1 Protein Expression}

ZR-75-1 and MCF-7 are estrogen receptor (ER)-positive human breast cancer cell lines, with the latter being a classic model system used to study estrogen-dependent tumorigenesis. The detection of endogenous ESE-1 in ZR-75-1 and MCF-7 cells makes these ideal cell lines to study the role of ESE-1 in maintaining tumorigenesis, and whether ESE-1 contributes to cellular survival, apoptosis and/or proliferation. To address these points, we used an shRNA approach to knockdown endogenous ESE-1 in ZR-75-1 and MCF-7 cells. In initial optimization studies of several shESE-1 constructs, we identified an shESE-1 construct (shESE-1), which targeted the ETS DBD of ESE-1, that optimally knocked down endogenous ESE-1 (data not shown). Using shESE-1, we first sought to establish the time course of ESE-1 knockdown 
by transiently transfecting ZR-75-1 cells with shESE-1 and preparing whole cell lysates $2,3,4$ and 5 days post-transfection (Fig. 3A). As a control, we transfected cells with an

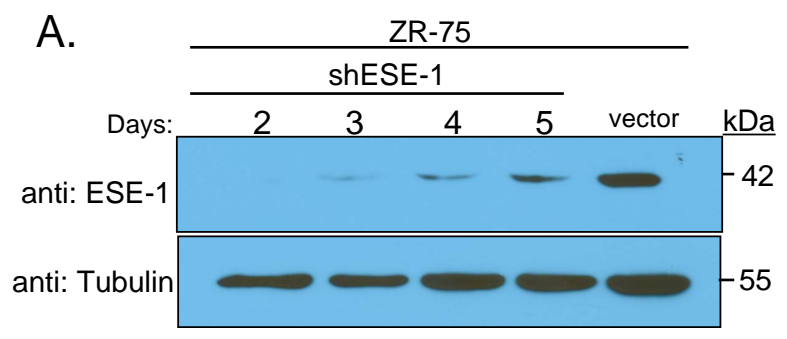

B.
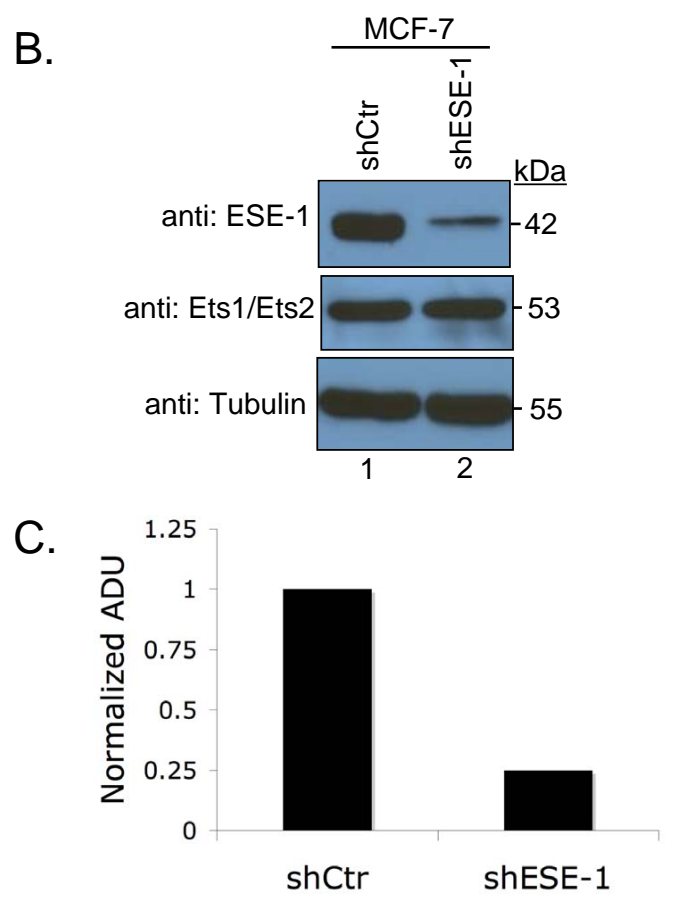

Fig. (3). shESE-1 knocks down endogenous ESE-1 protein in ZR-75-1 and MCF-7 cells in a specific and prolonged manner. A. Time-course of ESE-1 knockdown after transient shESE-1 transfection in ZR-75-1 cells. ZR-75-1 cells ( $\sim 60 \%$ confluent, 10 $\mathrm{cm}$ plate) were transiently transfected with shESE-1 (6.5:0.65 $\mu \mathrm{g}$; shESE1:pEGFP-C3), and lysates were harvested on days 2, 3, 4 and 5 post-transfection. The negative control lysate was generated 2 days after transfecting ZR-75-1 cells with pSuper/empty vector. Western blot of whole cell lysates $(100 \mu \mathrm{g})$ using the anti-ESE-1 mAB405 (1:1000) is shown in the top panel, with the same blot stripped and re-probed with tubulin antibody $(1: 10,000)$ and shown in the bottom panel. B. Specificity of shESE-1 knockdown in MCF7 cells. MCF-7 cells were transiently transfected with shCtr or shESE-1, harvested $48 \mathrm{~h}$ post-transfection and Western blot of 100 $\mu \mathrm{g}$ of whole cell extract with anti-ESE-1 mAB405 (1:1000) shown in the top panel. The middle panel shows a Western blot of $35 \mu \mathrm{g}$ of the same MCF-7 whole cell extracts, probed with anti-Ets1/Ets2 rabbit polyclonal antibody (1:10,000 dilution, Santa Cruz, c-275). The PVDF membrane shown in the top panel was washed and reprobed with anti-tubulin antibody $(1: 10,000)$, and the resultant Western blot is shown in the bottom panel. C. Arbitrary densitometry units (ADU) measuring the ESE-1 densitometry signal normalized against tubulin, with the shCtr signal set to 1 .
shRNA empty vector control (vector) and prepared whole cell lysates 2 days post-transfection (Fig. 3A). The whole cell lysates were then probed for ESE-1 and tubulin by Western blot analysis (Fig. 3A). These results show that compared to vector control, an almost complete knockdown of ESE-1 occurs by 2 days, with reduction of ESE-1 persisting up to 5 days post-transfection of shESE-1 (Fig. 3A). The tubulin control shows that a nearly equivalent amount of protein was loaded in each lane, indicating that differences in protein loading fail to explain the significant reduction in ESE-1 detected. A similar time course of shESE-1 knockdown was performed in MCF-7 cells and this study showed the same strong reduction of ESE- 1 by 2 days, and this level of inhibition persisted up to 5 days in MCF-7 (data not shown). Next, we sought to establish the specificity of ESE1 knockdown. As noted above, this shESE-1 targeted the ETS DBD, which is conserved amongst ETS proteins. Computational analysis of the shESE-1 target sequence revealed it to be unique to ESE-1, with our target sequence showing minimal similarity only to ETS-1/ETS-2 (with only 4 of 19 nt being identical for each). As a negative control (shCtr), we used an shRNA construct that also targeted the ESE-1 ETS DBD, but which in optimization studies failed to inhibit ESE-1 expression. We transiently transfected MCF-7 cells with shCtr and shESE-1 shRNA vectors, prepared whole cell lysates 2 days post-transfection, and probed for ESE-1, ETS$1 /$ ETS-2 and tubulin by Western blot analysis (Fig. 3B). This study reveals that the shCtr failed to inhibit endogenous ESE-1, while the shESE-1 vector resulted in knockdown of ESE-1 in MCF-7 cells. We quantitated this inhibition by normalizing ESE-1 expression to tubulin and found that ESE-1 expression is reduced $\sim 4$-fold in the shESE-1 cells compared to shCtr (Fig. 3C). Finally, to determine the specificity of shESE-1 knockdown, we performed Western blot analysis for both ETS-1 and ETS-2, using an antibody that recognizes both ETS factors. As shown in Fig. (3B), neither the shCtr nor shESE-1 affected the levels of ETS-1 plus ETS-2, affirming shESE-1's specificity to knockdown endogenous ESE-1, and that the shCtr failed to inhibit ESE-1, ETS-1 and ETS-2.

\section{Knockdown of ESE-1 Reduces the Colony-Forming Ability of MCF-7 and ZR-75-1 Cells}

In order to determine if ESE-1 was necessary for colony formation, MCF-7 and ZR-75-1 cells were each co-transfected with pEGFP-C3, to confer G418 resistance, and shCtr or shESE-1. Transfected cells were selected with G-418 for 14 days, resultant colonies were stained with Crystal violet, and counted by direct visualization (Figs. 4A and 4B). The MCF7 and ZR-75-1 cells transfected with shCtr yielded $\sim 27$ and $\sim 43$ colonies per plate, respectively, with ZR-75-1 colonies being larger than the MCF-7 colonies (Fig. 4A). Quantitation of triplicate colony formation assays indicated that only $\sim \operatorname{six}$ MCF-7 colonies formed in the presence of shESE-1, resulting in a 5.5-fold reduction in MCF-7 colony formation and only $\sim$ three ZR-75-1 colonies formed in the presence of shESE-1, resulting in a 13-fold reduction in ZR-75-1 colony formation (Fig. 4B). Of note, we show a 14-day selection with G418, since several attempts to generate stable ESE-1 knockdown cell lines resulted in very few, small colonies that failed to grow, thus making clonal expansion unsuccessful. Importantly, the similar inhibitory effect of ESE-1 


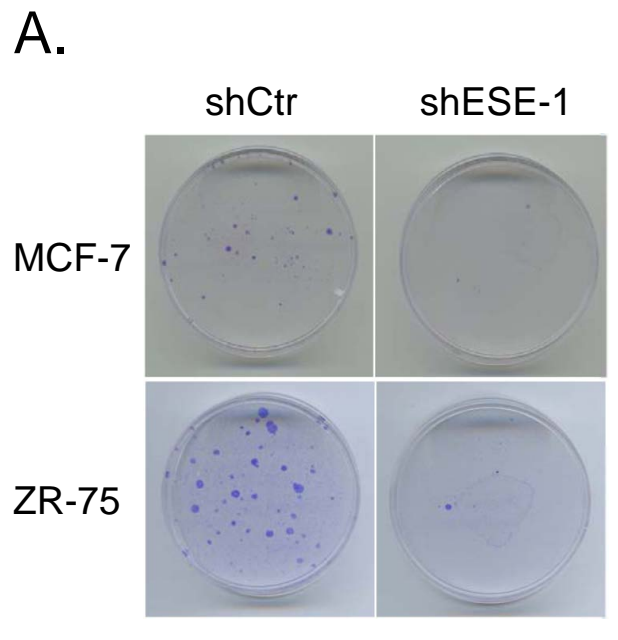

B.

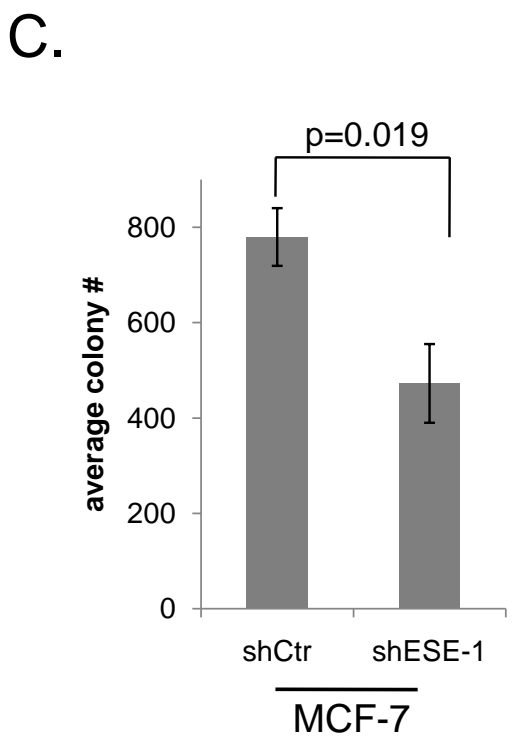

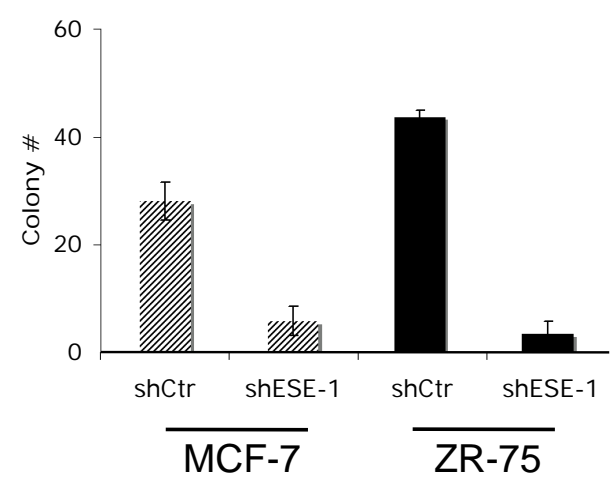

D.

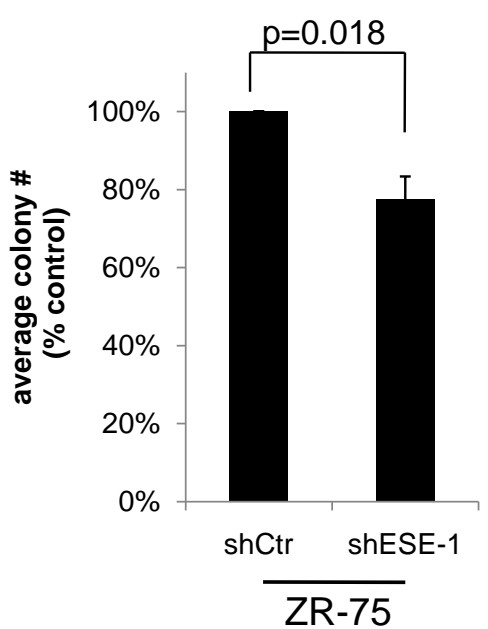

Fig. (4). ESE-1 knockdown abrogates MCF-7 and ZR-75-1 colony formation and diminishes soft agar colony formation. A. MCF-7 and ZR-75-1 colonies stained with Crystal violet. MCF-7 cells (top panels) or ZR-75-1 cells (bottom panels) were transfected with shCtr or shESE-1 (using different DNA preparations for the ZR-75-1 and MCF-7 cells), selected for 14 days with 500 ug/ml of G418, and resultant colonies were stained with Crystal violet. B. Direct visual quantitation of MCF-7 (grey bars) and ZR-75-1 (dark bars) colonies. Cells were transfected in triplicate and selected with G418, as above, and the resultant colonies were counted by visual inspection. The shESE-1mediated colony reduction in MCF-7 and ZR-75-1 cells is statistically significant to $p=0.008$ and $p=0.0006$, respectively, using the Student's t-test. C. Quantitation of MCF-7 soft agar colonies. MCF-7 cells were transiently transfected with shCtr or shESE-1, plated in soft agar and resulting colonies were counted after 21 days using imaging software. Data shown are average of 5 independent assays. D. Quantitation of ZR-75 soft agar colonies. Data shown are an average of 5 independent assays. Results from each experiment were normalized to shCtr and expressed as \% control.

knockdown on colony formation in two distinct breast cancer cell lines supports the critical role of ESE-1 in the growth of transformed mammary cells.

\section{Knockdown of ESE-1 Inhibits Anchorage Independent Growth of MCF-7 and ZR-75-1 Cells}

To further investigate the functional role of ESE-1 in the maintenance of tumorigenic phenotype, we evaluated the effects of ESE-1 knockdown on anchorage independent growth of MCF-7 and ZR-75-1 breast cancer cells using soft agar assays. To this end, we transiently transfected MCF-7 and ZR-75-1 cells with shCtr or shESE-1 vector DNAs, plated the cells in soft agar and after 14 days counted the colonies growing in an anchorage independent manner (Fig. 4C and 4D). Because in the transient transfection approach not all cells are transfected and ESE-1 expression is likely to re-appear at later time points, compared to G418 selection methods, the resulting colony number in the shESE-1 knockdown cells presented here is likely an overestimate. The graph in Fig. (4C) shows quantification of MCF-7 soft agar colonies derived from 5 independent experiments. MCF-7 cells transfected with shCtr generated on average $\sim 780$ colonies, whereas MCF-7 cells transfected with shESE-1 generated $\sim 470$, a $40 \%$ reduction in colony formation. The cloning efficiency, determined by dividing the number of 
colonies by the number of cells seeded, was on average $1.6 \%$ for shCtr-transfected MCF-7 cells and $0.9 \%$ for shESE-1 transfected MCF-7 cells. Colony formation in soft agar by ZR-75-1 cells was less efficient and less reproducible than by MCF-7 cells. Despite further optimization, the number of soft agar colonies formed by ZR-75-1 cells fluctuated from assay to assay, possibly because these cells were sensitive to slight changes in assay conditions. Nevertheless, we consistently observed that the shESE-1-transfected ZR-75-1 cells formed less colonies than the cells transfected with shCtr. For quantification, we normalized the colony number in each assay to shCtr $(100 \%)$. The graph in Fig. (4D) shows quantification of ZR-75-1 soft agar colonies, derived from 5 independent experiments. ZR-75-1 cells transfected with shESE1 formed on average $22 \%$ fewer colonies than the cells transfected with shCtr.

To address the possibility that the observed growthinhibitory effects of shESE-1 on MCF-7 cells are due to a nonspecific, off-target knockdown, we used another shESE1, labeled as shESE-1/ob4. As previously noted, in our initial studies testing of seven shESE-1 constructs, only one resulted in knockdown of ESE-1. Thus, to identify another shRNA construct, we tested an additional five constructs in a distinct vector backbone (pLKO.1), and identified shESE1/ob4 as one that resulted in measurable ESE-1 knockdown (Fig. 5). MCF-7 cells were transiently transfected with shGFP control or shESE-1/ob4, both in the pLKO.1 backbone, and whole cell lysates were analyzed by Western blotting. The results show that the level of endogenous ESE1 protein was partially inhibited by shESE-1/ob4 relative to control, and that the amount of protein loaded was equivalent in each lane (Fig. 5A). Quantitation of ESE-1 protein expressed, normalized to tubulin, shows that shESE-1/ob4 mediates a $27 \%$ knockdown (Fig. 5B). MCF-7 cells transfected with shGFP generated $\sim 1343$ colonies, whereas shESE1/ob4-transfected cells generated $\sim 874$ colonies, a $35 \%$ reduction in colony formation (Fig. 5C). While shESE-1/ob4 mediated only a partial inhibition of soft agar colony formation, the effect essentially matched the knockdown level of ESE-1. Taken together, these functional assays indicate that the effects of shESE-1 and shESE-1/ob4 are unlikely to be due to nonspecific off-target responses, and demonstrate that ESE-1 is required to maintain the transformed phenotype of MCF-7 cells.

\section{Reversion of the Transformed Phenotype is not due to Apoptosis}

Having demonstrated a reduction in colony formation and anchorage independent growth, we next sought to address the mechanism responsible for the reversion of the malignant phenotype. We first tested whether knockdown of ESE-1 in MCF-7 cells resulted in apoptosis. In order to address this point, MCF-7 cells were transiently transfected with empty vector, shCtr or shESE-1, and harvested at 48 and 72 hours. We tested for apoptosis by using two separate assays, DNA laddering (Fig. 6A) and caspase 3/7 analyses (Fig. 6B and 6C). The DNA laddering assay showed no DNA fragmentation at the 48-hour time point for vector control, indicating that the transfection method alone did not induce apoptosis (Fig. 6A, lane 1). Similarly, there was no DNA fragmentation at the 48 -hour time point in the shESE-1
A.
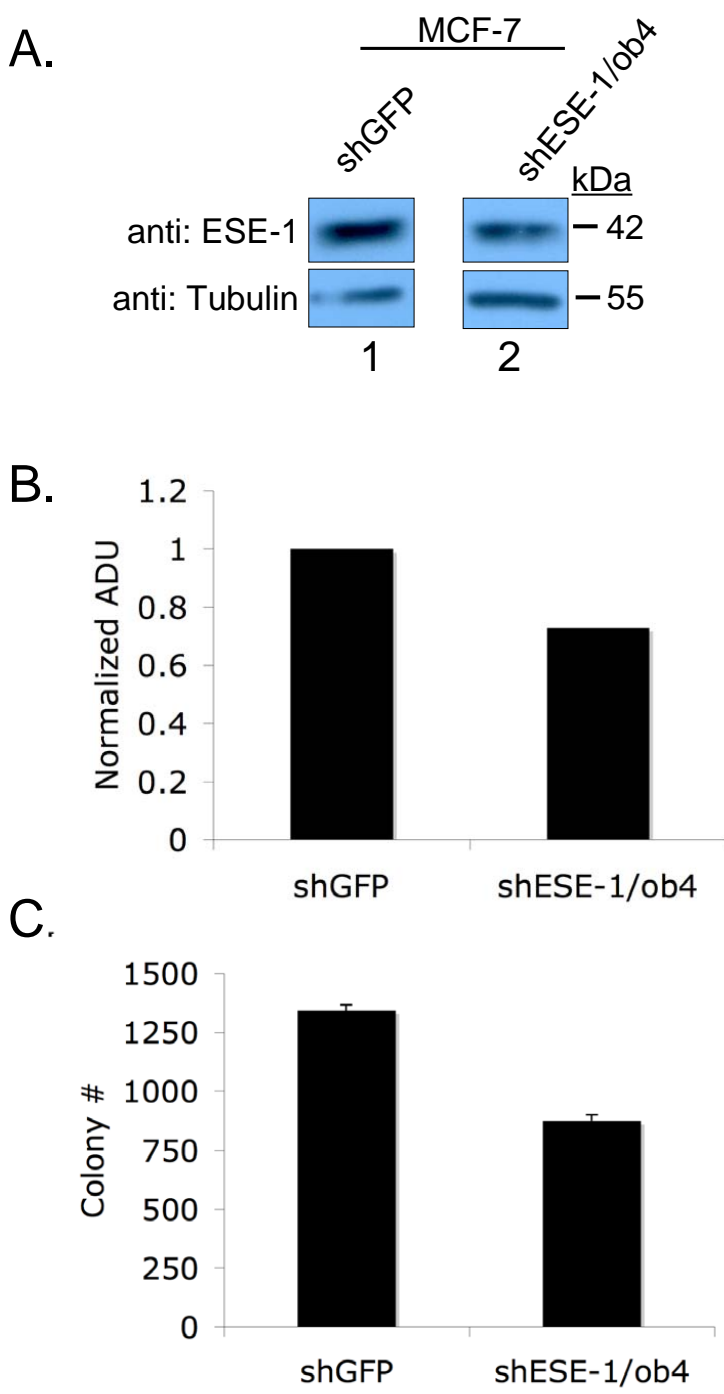

Fig. (5). shESE-1/ob4-mediated partial knockdown of endogenous ESE-1 protein correlates with the partial reduction in soft agar colony count in MCF-7 cells. A. ESE-1 Western blot. MCF-7 cells were transiently transfected with shGFP or shESE1/ob4, harvested $48 \mathrm{~h}$ post-transfection and Western blot of $50 \mu \mathrm{g}$ of whole cell extract with anti-ESE-1 mAB405 (1:1000) shown in the top panel. The PVDF membrane shown in the top panel was washed and re-probed with anti-tubulin antibody $(1: 10,000)$, and the resultant Western blot is shown in the bottom panel. B. Relative quantitation of ESE-1 expression. Arbitrary densitometry units (ADU) measuring the ESE-1 densitometry signal normalized against tubulin, with the shCtr signal set to 1. C. Quantitation of soft agar colonies. MCF-7 cells were transiently transfected with shGFP or shESE-1/ob4, plated in sextuplicate or triplicate and colonies were quantitated using the Metamorph imaging software (threshold 150-250 microns). shESE-1/ob4 mediated a $35 \%$ reduction in MCF-7 soft agar colony formation, which was significant to a $\mathrm{p}=4.4 \times 10^{-10}$ value, using the Student's t-test.

or shCtr knockdown lanes (Fig. 6A, lanes 2-3). At the 72hour time point there is minimal DNA fragmentation in the empty vector (Fig. 6A, lane 4) and shCtr (Fig. 6A, lane 5) controls, and no detectable fragmentation in the shESE-1 treated cells (Fig. 6A, lane 5). As a positive control, MCF-7 cells were treated with Trail plus cycloheximide, and these 

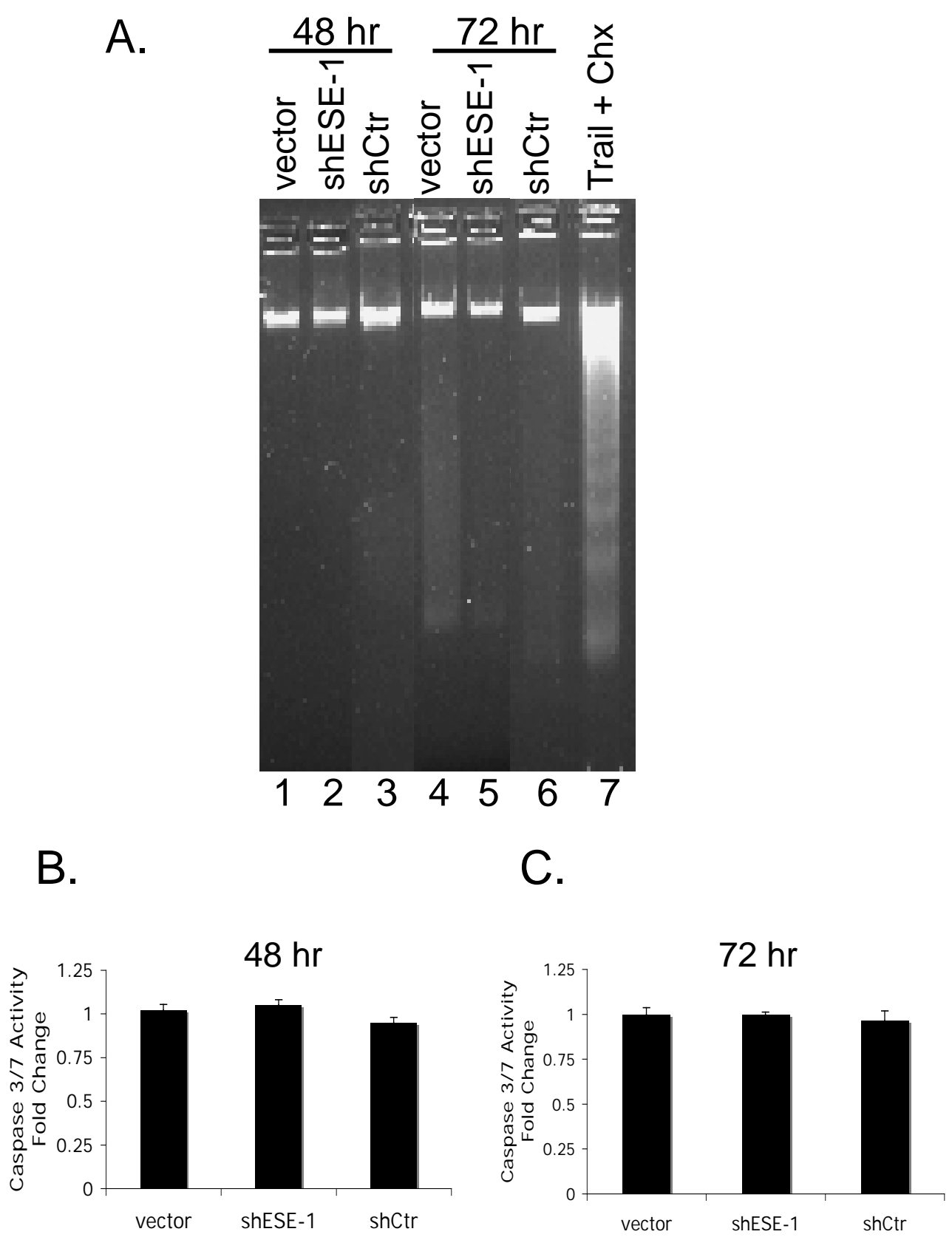

Fig. (6). Knockdown of ESE-1 does not induce DNA laddering or Caspase 3/7 activity. MCF-7 cells were transiently transfected with empty vector (lanes $1 \& 4$ ), shESE-1 (lanes $2 \&$ 5) and shCtr (lanes $3 \& 6$ ), and genomic DNA was isolated at 48 and $72 \mathrm{~h}$ post-transfection. Isolated DNA $(5 \mu \mathrm{g})$ was separated on a $1 \%$ agarose gel and stained with ethidium bromide. Positive control MCF-7 cells (lane 7$)$ were treated with $75 \mu \mathrm{g} / \mathrm{ml}$ of trail plus $1 \mu \mathrm{g} / \mathrm{ml}$ of cycloheximide for $24 \mathrm{~h}$, and DNA $(5 \mu \mathrm{g})$ was isolated and analyzed as above. B. and C. For the caspase assays MCF-7 cells were transiently transfected with empty, shESE-1 and shCtr DNAs in triplicate, and caspase 3/7 activity was measured at $48 \mathrm{~h}(\mathrm{~B})$ and $72 \mathrm{~h}(\mathrm{C})$ post-transfection. The capsase 3/7 activity of untransfected controls at 48 and $72 \mathrm{~h}$ was set to 1 , and the caspase $3 / 7$ activity of transfected cells was normalized to the untransfected value and expressed as fold-change.

cells display robust DNA fragmentation evincing apoptosis (Fig. 6A, lane 7). To further investigate apoptosis as a biological response to ESE-1 knockdown, we analyzed caspase 3 and/or 7 activation, using a luminescent enzyme activity assay (CaspaseGlo 3/7 Assay, Promega). With the data set to 1 for untransfected controls, these results show that there is no change in caspase $3 / 7$ activity in shESE- 1 transfected cells, compared to empty vector and shCtr transfected cells, at either the 48 or 72 hour time points (Figs. 6B and 6C). Having excluded apoptosis as the cellular mechanism responsible for the shESE-1-induced inhibition of colony formation, we next sought to determine whether shESE-1 affected MCF-7 cellular proliferation. 


\section{Reversion of the Transformed Phenotype is due to shESE-1-Mediated Inhibition of MCF-7 Cell Prolifera- tion}

The control of cellular proliferation is a key mechanism in the prevention of tumorigenicity and malignancy. ESE-1 has been shown to regulate promoter activity of the Her2/ $n e u$ and TGF- $\beta R I I$ genes [13, 24-27]. These two plasma membrane receptors contribute to the regulation of breast cancer cell growth and proliferation. ESE-1's transcriptional regulation of these receptors suggests that it has an important role in controlling cellular proliferation. In order to confirm ESE-1's role in maintaining cellular proliferation in MCF-7 transformed cells, MCF-7 cells were transiently transfected with shCtr or shESE-1, and cellular proliferation was determined at 2-, 4- and 6-days post-transfection by counting total viable cells (Fig. 7A) and 6-days post-transfection using an MTS assay (Fig. 7B). A representative total cell proliferation study, performed in duplicate, shows that shESE-1 cells display significantly reduced proliferation at each time point (Fig. 7A). At the start of the study, 50,000 cells were plated, with shCtr cells showing 82,500, 105,000 and 260,000 at 24- and 6-days post-transfection and the shESE-1 cells showing $31,000,23,500$ and 89,000 at the same time points. These results reveal a $62 \%, 78 \%$ and $66 \%$ reduction in cell proliferation at 2- 4- and 6-days, respectively, in the shESE1 knockdown cells compared to shCtr cells (Fig. 7A). Similar results were obtained using an MTS proliferation assay, which allowed us to perform 8 replicates in a 96-well format, and showed a 1.6 fold (or $\sim 39 \%$ ) reduction in MTS absorbance at 6-days post-transfection in the shESE-1 transiently transfected MCF-7 cells, compared to shCtr control cells (Fig. 7B). These data further demonstrate that ESE-1 is required for optimal MCF-7 cellular proliferation and reveal the mechanism by which ESE-1 contributes to the transformed phenotype.

\section{DISCUSSION}

The ETS transcription factor family is known to play a significant role in many cancers, with aberrant expression of ESE-1 being detected in nearly $50 \%$ of early human breast tumors. In addition, ectopically expressed ESE-1 has been shown to impart the transformed phenotype on ESE-1negative MCF-12A and MCF-10A nontransformed mammary epithelial cell lines $[15,20-22,28]$. While dominantnegative ETS approaches, which interfere with multiple ETS factors, have reversed the transformed phenotype in several breast cancer cell lines (NmuMG, MMT and BT20) [18, 19], here we show that the knockdown of a single ETS factor, ESE-1, has the same effect in MCF-7 and ZR-75-1 breast cancer cells. Importantly, we show that ESE-1 is required to maintain the transformed phenotype in MCF-7 and ZR-75-1 breast cancer cells, since shRNA-mediated ablation of endogenous ESE-1 protein resulted in decreased colony formation and anchorage-independent growth (Figs. 4-6). Furthermore, mechanistic studies, using two separate approaches to measure apoptosis and proliferation, revealed that ESE-1 does not modulate apoptosis in MCF-7 cells, but rather is required for their proliferation (Figs. 6 and 7). Taken together, this paper contributes novel insights to our understanding of the critical role of ESE-1 in maintaining cell
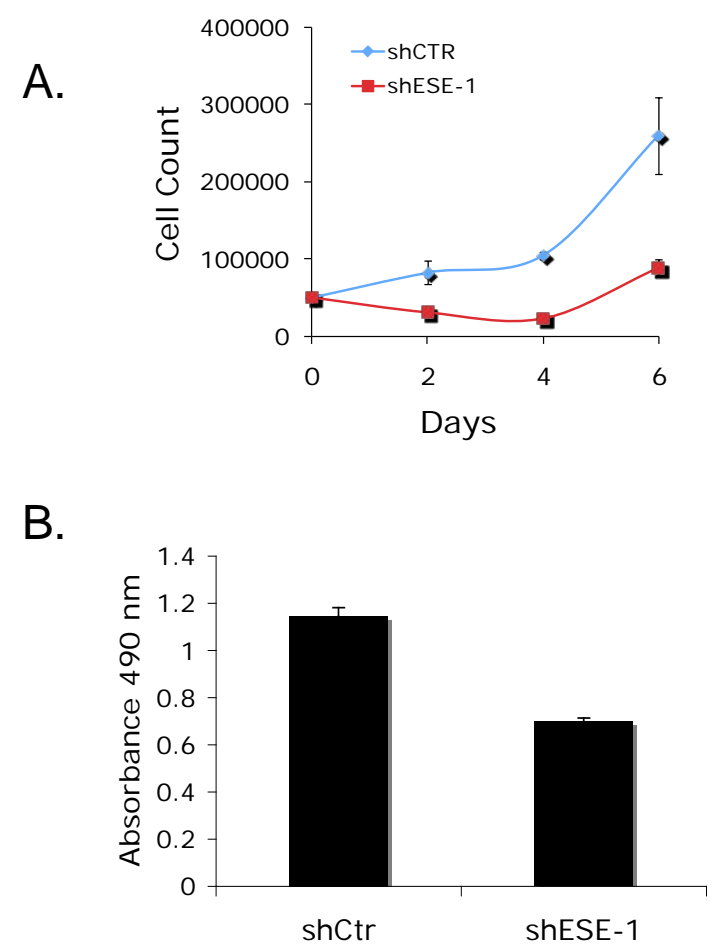

Fig. (7). Proliferation of MCF-7 cells is reduced with knockdown of ESE-1. A. Total cell counts over 6 days. MCF-7 cells were transiently transfected with shCtr (diamonds) or shESE-1 (squares), and $24 \mathrm{~h}$ post-transfection cells were counted and 50,000 seeded at time 0 . Cells were collected on days 2, 4, and 6 with $1 \mathrm{x}$ PBS/EDTA and counted using the Beckman Coulter Vi-cell. B. MTS proliferation assay at 6 days. MCF-7 cells were transiently transfected with shCtr or shESE-1, replicated eight times, and $24 \mathrm{~h}$ post-transfection cells were counted and 5000 cells were plated at time 0. Cells were grown for 6 days, harvested and MTS measured at $490 \mathrm{~nm}$, as in Methods. The difference in shCtr $v s$. shESE-1 was significant to $\mathrm{p}=1.67 \mathrm{E}-07$, using the Student's t-test.

transformation of mammary epithelial cells via regulation of cellular proliferation.

Protein expression and characterization studies of ESE-1 in breast cancer cell lines and tissues have been limited, in large part due to the lack of highly-specific anti-ESE-1 antibodies. Similar to our results shown here, most studies using Western blot analysis of breast cancer cell lines have reported that MCF-10A, MCF-12A and MDA-MB-231 cells typically do not express ESE-1, whereas MCF-7, ZR-75-1 and T47D cells do express it [13, 28-30]. However, unlike our results, one group reported ESE-1 protein expression in MCF-12A cells [13], and another group reported that T47D cells fail to express ESE-1 protein [30]. We have consistently not been able to detect ESE-1 in MCF-12A cells, using PCR to detect mRNA (Fig. 1B) [21] and Western blot, IHC or ICC analyses to detect protein (Figs. 1A and 2) [20]. Thus, one possibility to explain this discrepancy is that MCF-12A cells expressing ESE-1 have undergone spontaneous transformation, suggesting that MCF-12A cells may be poised to be easily transformed, requiring the use of low passage cells grown in defined media to avoid transforma- 
tion and possible activation of ESE-1 expression [21, 31]. Another discrepancy is that we show the level of ESE-1 protein to be MCF-7 = T47D $>$ ZR-75-1, whereas previous reports show MCF-7 > ZR-75-1 >> T47D [13, 29, 30]. This disparity could be explained by slight differences in growth conditions or subclone characteristics. Indeed, we have found ESE-1 expression to vary in certain T47D sublines (data not shown). While many more cell lines would have to be analyzed to reach a rigorous conclusion, it is noteworthy that MCF-10A, MCF-12A and MDA-MB-231 cells, which fail to express Her2/neu, also fail to express ESE-1; whereas ZR-75-1, T47D and MCF-7, which express detectable to low to minimal levels of Her2/neu, respectively, do express ESE1. Finally, we show that ESE-1 mRNA levels generally correlate with protein data in the mammary cell lines studied here, with T47D and MDA-MB-231 showing a slight discordance between the mRNA and protein levels (Fig. 1).

ESE-1 contains several functional NLS and NES signals $[20,32]$, suggesting that ESE-1 shuttles between the nuclear and cytoplasmic compartments. We and others have demonstrated that transient transfection and adenoviral transduction studies consistently show nuclear localization of ESE-1/Elf-3 and we have used such transient ESE-1 expression assays to map the transcriptional properties and sites of co-factor interactions of ESE-1/Elf-3 as a nuclear effector [15, 20, 29, 32-35]. Here, using MAb405 in ICC studies, we show that endogenous ESE-1 is detected in the nucleus in MCF-7, T47D and ZR-75-1 human mammary epithelial cancer cells (Fig. 2). However, several IHC studies of endogenous ESE-1 show cytoplasmic \pm nuclear localization in T47D and ZR75-1 cell lines [28], and mammary, retinal pigment and synovial epithelial cells [13, 20, 29, 36-38]. Thus, the differences in subcellular localization of ESE-1 noted in these various studies may be due to the different experimental conditions, different antibodies used for detection, and different types of assays: e.g., IHC vs. ICC, transient vs. stable, ectopic vs. endogenous, in vitro vs. in vivo, and nontransformed $v s$. transformed. Nevertheless, in combination, these studies reveal that ESE-1 can be detected in the nuclear and/or cytoplasmic compartments.

Previously, we have shown that stably expressed HAESE-1 or GFP-ESE-1 initiates and imposes the transformed phenotype upon nontransformed, ESE-1-negative MCF-10A and MCF-12A cells [20, 21], and that cytoplasmic localization of a 40-AA SAR domain is necessary and sufficient to mediate this effect [20]. Consistent with this conclusion, PAK-1-mediated phosphorylation of the SAR domain of exogenous ESE-1 in the cytoplasm modulates the stability and transformation potency of ESE-1 in ZR-75-1 cells [28]. Here we show that ESE-1 knockdown, in transformed MCF7 and ZR-75-1 cells that express endogenous ESE-1, reverses their transformed properties. We used an additional, distinct shESE-1 construct (shESE-1/ob4) in MCF-7 cells to address any nonspecific effects using shESE-1 to knockdown ESE-1. Although the ESE-1 knockdown and colony inhibition was less with the shESE-1/ob4 construct compared to shESE-1 $(\sim 30 \% v s . \sim 40 \%)$, the reduction in soft agar colony formation was correlated with the level of endogenous ESE-1 protein (Figs. 4 and 5). Thus together, these two knockdown studies confirm that the reversal of the transformed phenotype is due to reduction of ESE-1, rather than any nonspecific effects of shRNA expression.
Moreover, we demonstrate, by ICC, that ESE-1 is localized to the nucleus in MCF-7, T47D and ZR-75-1 cells, indicating that the ability of ESE-1 to maintain the transformed phenotype requires its role as a nuclear transcription factor. Of note, we have previously demonstrated that GFPESE-1 targeted to the nucleus of nontransformed MCF-10A and MCF-12A cells induces apoptosis, whereas transformed T47D and Sk-Br-3 cells tolerate nuclear expression of exogenous ESE-1 without inducing apoptosis, possibly because anti-apoptotic pathways have been up-regulated in these transformed cells [20]. Taken together, these data suggest that ESE-1 initiates transformation in ESE-1-negative mammary epithelial cells via a cytoplasmic- and PAK-1-dependent mechanism, but once fully transformed, these cells require the nuclear transcription properties of ESE-1 to maintain the transformed phenotype.

ETS factors have been associated with a number of malignancies, particularly in the mammary gland and prostate [1]. The large redundancy of ETS factor genes in humans $(\sim 27)$ has required dominant-negative ETS approaches, whereby function of multiple ETS factors is blocked. This dominant-negative method has validated that ETS factors are required for mammary cell transformation [1]; however, such studies have failed to identify whether an individual ETS factor is sufficient to maintain the transformed phenotype. A key significance of this report is that we use shRNA technology to knockdown a single ETS factor, ESE-1, and this loss of function study supports that ESE-1 is necessary to maintain the transformed phenotypes in MCF-7 and ZR-75-1 cells. Our previous gain of function studies support that ESE-1 can be sufficient for transformation [21]. Thus, the clinical significance is that ESE-1 provides a novel single-molecule target for breast cancer therapy. The optimal ESE-1 19-nt target sequence (shESE-1) maps to the DBD. While BLAST searches with this 19-nt sequence only identified ESE-1, manual analyses of ETS DBD sequences revealed that ETS- 1 and ETS-2 showed the closest similarity, with only 4/19 matches. While MCF-7 cells express low levels of ETS-2 and minimal amounts of ETS-1, this level of mismatches is very unlikely to allow the ESE-1 shRNA to target either ETS-1 or ETS-2 [39, 40]. Nevertheless, we performed Western blot analysis and demonstrated specificity for knockdown of ESE-1, since there was no change in the level of what is likely to be ETS-2 (Fig. 3B). Relevant to this report is the Ets2 knock-out study revealing that transgenic mice expressing mammary-targeted polyoma virus middle $\mathrm{T}$ oncogene crossed to heterozygous female mice carrying only one wild-type Ets 2 allele, resulted in smaller tumors compared to crosses with wild-type controls [41]. Further analysis revealed that Ets2 functions in the stromal compartment to regulate mammary epithelial tumor growth [42]. The transgenic papers show a $\sim 50 \%$ reduction in mouse mammary tumor size, due to the stromal effects of Ets2, whereas we show an $\sim 80-90 \%$ reduction in colony formation in ZR-75-1 and MCF-7 cells stably expressing shESE-1 (Fig. 4A and B) and a $\sim 20$ and $\sim 40 \%$ reduction in soft agar colony numbers in ZR-75-1 and MCF7 cells, respectively, transiently expressing the shESE-1 (Fig. 4C and D). Indeed, the colonies growing in G418-selected MCF-7 cells, with stable knockdown of ESE-1, consistently failed to expand, underscoring that ESE-1, when more fully ablated, has a dominant growth and/or survival inhibitory 
effect in mammary epithelial cells. In combination, these data reveal that specific, individual ETS factors have critical roles in mammary carcinogenesis, but in distinct tissue compartments, with Ets2 functioning in the stroma and ESE1 functioning in the mammary epithelial cell.

Having demonstrated that ESE-1 is required to maintain the transformed phenotype of MCF-7 cells, we proceeded to mechanistic studies evaluating whether ESE-1 knockdown induced apoptosis or affected cell proliferation. Specifically, we used both DNA laddering and caspase 3/7 cleavage assays, in order to rigorously assess whether ESE-1 knockdown caused apoptosis, and the data show that ESE-1 knockdown does not induce MCF-7 apoptosis (Fig. 6). Instead, two separate experimental approaches, total cell counts and MTS assay, revealed that ESE-1 knockdown has a key effect on MCF-7 cell proliferation (Fig. 7). Again, these studies required transient transfection of shESE-1 in order to expand the cells over the 6-day assay period, and despite the fact that not all cells take up plasmid DNA, shESE-1 knockdown resulted in a $66 \%$ and $39 \%$ inhibition of proliferation in the total cell count and MTS assays, respectively, at 6 days (Fig. 7). Certain ETS proteins control cell proliferation by inducing growth factors and/or their receptors, with ESE-1 shown to trans-activate HER2/neu [1, $5,15,35,43,44]$. Indeed, heregulin/HER2, EGF and serum induce ESE-1 promoter activity in Sk-Br-3 human breast cancer cells. Furthermore, lactogenic competency induced in murine HC-11 mammary cells by dexamethasone, insulin and prolactin, also increased ESE-1 mRNA expression [13]. These findings suggest that a forward autocrine regulatory loop may exist between ESE-1 and growth factors, such as HER2/neu, and that such a regulatory loop contributes to the regulation of cell proliferation, and ultimately transformation, by ESE-1.

\section{CONCLUSION}

Although an increasing number of reports have underscored the important role of ETS factors in human breast cancer, the exact role of any single ETS factor in maintaining the transformed phenotype in human breast cancer cell lines remains undefined. This report establishes that the knockdown of a single ETS factor, ESE-1, is sufficient to reverse the transformed phenotype of MCF-7 and ZR-75-1 breast cancer cell lines and demonstrates that ESE-1 is required for cellular proliferation. Taken together, this paper contributes novel insights to our understanding of the critical role of ESE-1 in maintaining the transformed state in breast cancer, providing a novel single-point target for therapy.

\section{ACKNOWLEDGEMENTS}

The authors wish to thank Drs. Heide Ford and Pepper Schedin for critical review of the manuscript. We thank members of the Gutierrez-Hartmann laboratory for their helpful comments and suggestions. We also thank Dr. Robert Winn for advice in optimizing the soft agar assay and Dr. Raphael Nemenoff for use of the Metamorph program. We used DNA Sequencing, Tissue Culture, Imaging and Quantitative PCR Core Facilities of the Colorado Comprehensive Cancer Center (supported by grant NIH P30 CA 46934). We acknowledge the following grant support, including: NIH
F31 GM 72203 to DMW, DOD BCRP W81XWH-05-1-0352

to JP, UNCF-Pfizer and ACS Postdoctoral Fellowship Awards to MSG, and DOD BCRP W81XWH-06-1-0502 to AGH.

\section{REFERENCES}

[1] Gutierrez-Hartmann A, Duval DL, Bradford AP. ETS transcription factors in endocrine systems. Trends Endocrinol Metab 2007; 18 : 150-8.

[2] Oikawa T, Yamada T. Molecular biology of the Ets family of transcription factors. Gene 2003; 303: 11-34.

[3] Sharrocks AD. The ETS-domain transcription factor family. Nat Rev Mol Cell Biol 2001; 2: 827-37.

[4] Wasylyk B, Hagman J, Gutierrez-Hartmann A. Ets transcription factors: nuclear effectors of the Ras/MAP kinase signaling pathway. Trends Biochem Sci 1998; 23: 213-6.

[5] Seth A, Watson DK. ETS transcription factors and their emerging roles in human cancer. Eur J Cancer 2005; 41: 2462-78.

[6] Tomlins SA, Rhodes DR, Perner S, et al. Recurrent fusion of TMPRSS2 and ETS transcription factor genes in prostate cancer. Science 2005; 310: 644-8.

[7] Andreoli J, Jang S-I, Chung E, Coticchia C, Steinert P, Markova N. The expression of a novel, epithelium-specific ets transcription factor is restricted to the most differentiated layers in the epidermis. Nucleic Acids Res 1997; 25: 4287-95.

[8] Feldman RJ, Sementchenko VI, Watson DK. The epithelialspecific Ets factors occupy a unique position in defining epithelial proliferation, differentiation and carcinogenesis. Anticancer Res 2003; 23: 2125-31.

[9] Oettgen $\mathrm{P}$, Alani $\mathrm{R}$, Barcinski $\mathrm{M}$, et al. Isolation and characterization of a novel epithelium-specific transcription factor, ESE1, a member of the ets family. Mol Cell Biol 1997; 17: 4419-33.

[10] Tymms M, Ng A, Thomas R, et al. A novel epithelial-expressed ETS gene, ELF3: Human and murine cDNA sequences, murine genomic organization, human mapping to 1q.32.2 and expression in tissues and cancer. Oncogene 1997; 15: 2449-62.

[11] Neve R, Chang C-H, Scott G, et al. The epithelium-specific Ets transcription factor ESX is associated with mammary gland development and involution. FASEB J 1998; 12: 1541-50.

[12] Chang CH, Scott GK, Kuo WL, et al. ESX: a structurally unique Ets overexpressed early during human breast tumorigenesis. Oncogene 1997; 14: 1617-22.

[13] Neve RM, Ylstra B, Chang CH, Albertson DG, Benz CC. ErbB2 activation of ESX gene expression. Oncogene 2002; 21: 3934-8.

[14] Benz C, O'Hagan R, Richter B, et al. HER2/Neu and the Ets transcription activator PEA3 are coordinatley upregulated in human breast cancer. Oncogene 1997; 15: 1513-25.

[15] Eckel KL, Tentler JJ, Cappetta GJ, Diamond SE, GutierrezHartmann A. The epithelial-specific ETS transcription factor ESX/ESE-1/Elf-3 modulates breast cancer-associated gene expression. DNA Cell Biol 2003; 22: 79-94.

[16] O'Hagan R, Hassell JA. The PEA3 Ets transcription factor is a downstream target of the HER2/Neu receptor tyrosine kinase. Oncogene 1998; 16: 301-10.

[17] Galang CK, Muller WJ, Foos G, Oshima RG, Hauser CA. Changes in the expression of many Ets family transcription factors and of potential target genes in normal mammary tissue and tumors. J Biol Chem 2004; 279: 11281-92.

[18] Delannoy-Courdent A, Mattot V, Fafeur V, et al. The expression of Ets-1 transcription factor lacking its activation domain decreases uPA proteolytic activity and cell motility, and impairs normal tubulogenesis and cancerous scattering in mammary epithelial cells. J Cell Sci 1998; 111: 1521-34.

[19] Sapi E, Flick M, Rodov S, Kacinski B. Ets-2 transdominant mutant abolishes anchorage-independent growth and macropahgestimulating factor-stimulated invasion by BT20 breast carcinoma cells. Cancer Res 1998; 58: 1027-33.

[20] Prescott J, Koto K, Singh M, Gutierrez-Hartmann A. The ETS transcription factor ESE-1 transforms MCF-12A human mammary epithelial cells via a novel cytoplasmic mechanism. Mol Cell Biol 2004; 24: 5548-64.

[21] Schedin P, Eckel-Mahan K, McDaniel S, et al. ESX induces transformation and functional epithelial to mesenchymal transition 
in MCF-12A mammary epithelial cells. Oncogene 2004; 23: 176679.

[22] Witt AE, Hines LM, Collins NL, et al. Functional proteomics approach to investigate the biological activities of cDNAs implicated in breast cancer. J Proteome Res 2006; 5: 599-610.

[23] Haugen BR, Larson LL, Pugazhenthi U, et al. Retinoic acid and retinoid $\mathrm{X}$ receptors are differentially expressed in thyroid cancer and thyroid carcinoma cell lines and predict response to treatment with retinoids. J Clin Endocrinol Metab 2004; 89: 272-80.

[24] Chang C, Scott G, Kuo W, et al. ESX: A structurally unique Ets overexpressed early during human breast tumorigenesis. Oncogene 1997; 14: 1617-22.

[25] Choi S-G, Yi Y, Kim Y-S, et al. A novel Ets-related transcription factor, ERT/ESX/ESE-1, regulates expression of the transforming growth factor- $\beta$ type II receptor. J Biol Chem 1998; 273: 110-7.

[26] Kim JH, Wilder PJ, Hou J, Nowling T, Rizzino A. Activation of the murine type II transforming growth factor-beta receptor gene: up-regulation and function of the transcription factor Elf-3/Ert/Esx/Ese-1. J Biol Chem 2002; 277: 17520-30.

[27] Park SH, Kim YS, Park BK, Hougaard S, Kim SJ. Sequencespecific enhancer binding protein is responsible for the differential expression of ERT/ESX/ELF-3/ESE-1/jen gene in human gastric cancer cell lines: Implication for the loss of TGF-beta type II receptor expression. Oncogene 2001; 20: 1235-45.

[28] Manavathi B, Rayala SK, Kumar R. Phosphorylation-dependent regulation of stability and transforming potential of ETS transcriptional factor ESE-1 by p21-activated kinase 1. J Biol Chem 2007; 282: 19820-30.

[29] Chang C-H, Scott GK, Baldwin NA, Benz CC. Exon 4-encoded acidic domain in the epithelium-restricted Ets factor, ESX, confers potent transactivating capacity and binds to TATA-binding protein (TBP). Oncogene 1999; 18: 3682-95.

[30] He J, Pan Y, Hu J, Albarracin C, Wu Y, Dai JL. Profile of Ets gene expression in human breast carcinoma. Cancer Biol Ther 2007; 6: 76-82.

[31] Paine TM, Soule HD, Pauley RJ, Dawson PJ. Characterization of epithelial phenotypes in mortal and immortal human breast cells. Int J Cancer 1992; 50(3): 463-73.

[32] Do HJ, Song H, Yang HM, et al. Identification of multiple nuclear localization signals in murine Elf3, an ETS transcription factor. FEBS Lett 2006; 580: 1865-71.
[33] Choi Y, Asada S, Uesugi M. Divergent hTAFII31-binding motifs hidden in activation domains. J Biol Chem 2000; 275: 15912-6.

[34] Wang H, Fang R, Cho JY, Libermann TA, Oettgen P. Positive and negative modulation of the transcriptional activity of the ETS factor ESE-1 through interaction with p300, CREB-binding protein, and $\mathrm{Ku}$ 70/86. J Biol Chem 2004; 279: 25241-50.

[35] Asada S, Choi Y, Yamada M, et al. External control of Her2 expression and cancer cell growth by targeting a Ras-linked coactivator. Proc Natl Acad Sci USA 2002; 99: 12747-52.

[36] Brown C, Gaspar J, Pettit A, et al. ESE-1 is a novel transcriptional mediator of angiopoietin-1 expression in the setting of inflammation. J Biol Chem 2004; 279: 12794-803.

[37] Grall F, Gu X, Tan L, et al. Responses to the proinflammatory cytokines interleukin-1 and tumor necrosis factor alpha in cells derived from rheumatoid synovium and other joint tissues involve nuclear factor kappaB-mediated induction of the Ets transcription factor ESE-1. Arthritis Rheum 2003; 48: 1249-60.

[38] Jobling AI, Fang Z, Koleski D, Tymms MJ. Expression of the ETS transcription factor ELF3 in the retinal pigment epithelium. Invest Ophthalmol Vis Sci 2002; 43: 3530-7.

[39] Barrett JM, Puglia MA, Singh G, Tozer RG. Expression of Etsrelated transcription factors and matrix metalloproteinase genes in human breast cancer cells. Breast Cancer Res Treat 2002; 72: 22732 .

[40] Lindemann RK, Braig M, Hauser CA, Nordheim A, Dittmer J. Ets2 and protein kinase $\mathrm{C}$ epsilon are important regulators of parathyroid hormone-related protein expression in MCF-7 breast cancer cells. Biochem J 2003; 372: 787-97.

[41] Neznanov N, Man A, Yamamoto H, Hauser C, Cardiff R, Oshima R. A single targeted Ets2 allele restricts development of mammary tumors in transgenic mice. Cancer Res 1999; 59: 4242-6.

[42] Man AK, Young LJ, Tynan JA, et al. Ets2-dependent stromal regulation of mouse mammary tumors. Mol Cell Biol 2003; 23: 8614-25.

[43] Scott GK, Chang CH, Erny KM, et al. Ets regulation of the erbB2 promoter. Oncogene 2000; 19: 6490-502.

[44] Chiang SY, Burli RW, Benz CC, et al. Targeting the ets binding site of the HER2/neu promoter with pyrrole-imidazole polyamides. J Biol Chem 2000; 275: 24246-54.

(C) Walker et al.; Licensee Bentham Open.

This is an open access article licensed under the terms of the Creative Commons Attribution Non-Commercial License (http://creativecommons.org/licenses/by$\mathrm{nc} / 3.0 /$ ), which permits unrestricted, non-commercial use, distribution and reproduction in any medium, provided the work is properly cited. 